\title{
Einen Schritt vor, zwei Schritte zurück
}

Das neue Erwachsenenschutzrecht enthält psychiatriespezifische Bestimmungen zur Fürsorgerischen Unterbringung sowie zu stationären und ambulanten Zwangsmassnahmen. Es schafft Sonderrecht im Bereich der Patientenverfügung, deren Wirksamkeit im Rahmen einer Fürsorgerischen Unterbringung im Vergleich mit den Vorschriften zur Somatik abgeschwächt wird.

René Bridler

Korrespondenz:

Dr. med. René Bridler M.H.A. Ärztlicher Direktor

Sanatorium Kilchberg AG

Alte Landstrasse 70

CH-8802 Kilchberg

Tel. 0447164265

Fax 0447164343

r.bridler[at]

sanatorium-kilchberg.ch
Man wird sich seinen eigenen gesunden Menschenverstand nicht dadurch beweisen können, dass man seinen Nachbarn einsperrt.

(Dostojewski, zit. nach Foucault)

\section{Einleitung}

Am 1. Januar 2013 trat das neue Erwachsenenschutzrecht (ESR) als Teil des Schweizerischen Zivilgesetzbuches (ZGB) in Kraft [1]. Wesentliche übergeordnete Revisionsanliegen waren - neben der Behördenprofessionalisierung - die Förderung des Selbstbestimmungsrechts, die Stärkung der familiären Solidarität sowie ein umfassender Schutz urteilsunfähiger Personen [2].

Die nachfolgenden Überlegungen beschränken sich auf die neuen Bestimmungen zur Psychiatrie, namentlich zur Patientenverfügung, zu deren Position im Rahmen einer Fürsorgerischen Unterbringung (FU) sowie zu den vorgesehenen Zwangsmassnahmen.

\section{Die Patientenverfügung}

Die Patientenverfügung ist das Instrument par excellence, das Selbstbestimmtheit bei ärztlichen und pflegerischen Interventionen sicherstellt. Mit einer solchen Anordnung (Art. 370-373 ZGB) kann jede urteilsfähige Person festlegen, welche medizinische Massnahmen sie im Fall einer zukünftigen Urteilsunfähigkeit gutheisst oder ablehnt. Sie kann zudem eine natürliche Person bezeichnen, die sich im Falle der Urteilsunfähigkeit mit den Fachleuten bespricht und stellvertretend entscheidet. Die Verankerung der Patientenverfügung im neuen ESR trägt dem gesellschaftlichen Empfinden Rechnung, wonach erkrankte Personen und medizinische Fachleute heute einander eher auf einer partnerschaftlichen Ebene als im traditionellen Rollenverständnis begegnen.

\section{Verbindlichkeit von Patientenverfügungen}

Fachleute sind neu verpflichtet, eine rechtskonforme Patientenverfügung zu befolgen, auch wenn sie Anordnungen enthält, die den Behandelnden

\section{Résumé}

Le nouveau droit de la protection de l'adulte contient des dispositions spécifiques psychiatriques concernant le placement à des fins d'assistance et traitement sans consentement dans le cadre hospitalier et ambulatoire. II constitue une dérogation des directives anticipées du patient qui atténue leur application dans le cas d'un placement à des fins d'assistance par rapport aux directives concernant les cas somatiques. Le texte révisé du code civil sera présenté sommairement et commenté en ayant recours à la littérature spécialisée disponible.

unpassend erscheinen [3]. Eine gültige Verfügung entfaltet grundsätzlich dieselbe Wirkung wie eine Jetzt-für-jetzt-Erklärung der urteilsfähigen Person und bedarf nicht der «objektiv-wissenschaftlichen» Vernünftigkeit [4]. Die gesetzgeberisch gewollte hohe Durchschlagskraft der Patientenverfügungen ist dennoch bemerkenswert. Zum einen ist es der Person freigestellt, sich bei der Erstellung des Dokumentes fachlich beraten zu lassen oder nicht [5]. Im Weiteren verzichtet das Gesetz auf semantische oder inhaltliche Vorschriften, die Anordnungen können allgemein gehalten oder sehr präzis formuliert sein. Und schliesslich nehmen Verfügungen für den somatischen Bereich mitunter Jahrzehnte entfernte, niemals zuvor erfahrbare existentielle Situationen in der Lebensendphase vorweg [6]; dieser Umstand macht sie spekulativ.

\section{Psychiatrische Patientenverfügungen}

Psychiatrische Patientenverfügungen sind in der Schweiz noch wenig verbreitet. Sie erteilen Anweisungen für Situationen, in denen aufgrund einer 


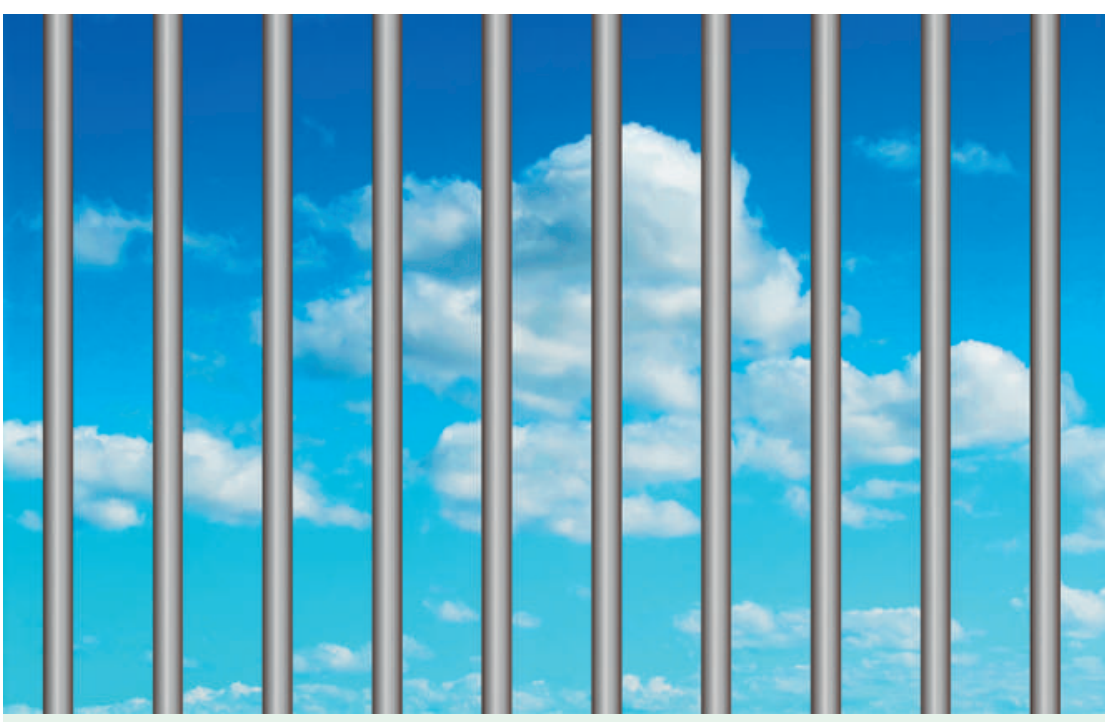

Neu beim Erwachsenenschutzrecht: Bei Fürsorgerischer Unterbringung kann der Chefarzt medizinische Massnahmen auch ohne Zustimmung anordnen.

schweren psychischen Krankheitsepisode vorübergehend Urteilsunfähigkeit eintritt und die Person nicht mehr in der Lage ist, sich ihren überdauernden Wünschen gemäss zu verhalten oder verständlich mitzuteilen [7]. Aus diesem Zustand können Hilflosigkeit und Angst, aber auch Stigmatisierung und nachhaltige Traumatisierung erwachsen - ganz besonders, wenn seitens des Psychiatriesystems Zwangsmassnahmen zur Anwendung gelangen [8,9]. Vor diesem Hintergrund besteht die grosse Stärke psychiatrischer Verfügungen darin, dass sie nicht spekulativ sind, sondern auf erlebter Krankheits-, Unterbringungs- und Therapieerfahrung beruhen.

Ein Blick in die wissenschaftliche Literatur zeigt, dass Psychiatrieerfahrene - entgegen den Befürchtungen der Fachleute - Patientenverfügungen fast nie zur Ablehnung jeglicher Behandlung verwenden. Stattdessen bevorzugen sie gewisse Kliniken, bestimmte Psychopharmaka und entwerfen realistische Therapieszenarien, die sich in Übereinstimmung mit Guidelines und den vor Ort üblichen Behandlungsmethoden befinden [10-13].

\section{Unverbindlichkeit von psychiatrischen Patientenverfügungen}

Im Gegensatz zur Regelung bei somatischen Erkrankungen sind psychiatrische Verfügungen unter den Bedingungen einer FU nicht verpflichtend, sondern müssen bei der Behandlung nur berücksichtigt werden; sie verlieren in diesem Rechtskontext die ihnen anderweitig zugestandene Verbindlichkeit (Art. 433 und 434 ZGB) [14]. Der Gesetzgeber beabsichtigt mit dieser Massnahme, eine Therapieverweigerung a priori zu unterbinden und die Durchsetzung des Behandlungsauftrags zu gewährleisten [15] (der unter FU-Bedingungen per definitionem ein staatlicher ist). Abgesehen von rechtsstaatlichen Bedenken, erscheint die Einführung von Sonderrecht für
Psychiatriepatienten aus fachlicher Perspektive als willkürlich, da psychiatrische Verfügungen auf persönlicher Krankheitserfahrung beruhen und durchaus realistisch ausgestaltet sind (s. oben).

\section{Stationäre Zwangsmassnahmen unter FU}

Die Bestimmungen zur FU basieren weitgehend auf den gemäss Gesetzgeber «bewährten» Regelungen zur Fürsorgerischen Freiheitsentziehung (FFE). Da im alten Gesetz eine FFE Zwangsmassnahmen nicht mit einschloss, bestanden kantonale Regelungen, was zu einer heterogenen Rechtslage und zu Kritik führte [2]. Bei dieser Kritik wird allerdings übersehen, dass Kantone auch die Möglichkeit hatten, Zwangsmassnahmen zu verbieten und stattdessen andere Massnahmen zu favorisieren. Diese «Gesetzeslücke» wurde nun geschlossen, neu können der Chefarzt oder die Chefärztin unter FU-Bedingungen medizinische Massnahmen ohne Zustimmung anordnen (Art. 434 i.V.m. Art. 433 ZGB).

\section{Ambulante Zwangsmassnahmen}

Neuland betritt das Erwachsenenschutzrecht, indem es die Kantone zur Regelung der Nachbetreuung nach einer FU verpflichtet und ihnen auch die Möglichkeit zur Anordnung von FU-unabhängigen ambulanten Massnahmen einräumt (Art. 437 ZGB). Obwohl es sich in erster Linie um freiwillige Auflagen zur Lebensführung, zur Beschäftigung oder zur Wahrnehmung einer Therapie handelt, steht es den Kantonen frei, auch Rechtsgrundlagen für den zwangsweisen Vollzug zu schaffen [16]. Während viele Kantone darauf verzichten, sieht der Kanton Aargau polizeiliche Zuführungen (§67p EG ZGB, Kanton AG) und der Kanton Schaffhausen sogar die zwangsweise Vollstreckung einer angeordneten Massnahme vor (Art. 60 EG KESR, Kanton SH).

Obwohl sich ambulante Zwangsmassnahmen international, vor allem in angelsächsischen Ländern, bei Gesundheitsbehörden einer steigenden Akzeptanz erfreuen, fehlt der empirische Wirksamkeitsnachweis. Die wenigen kontrollierten Studien mit prospektivem Design zeigten keine Vorteile im Vergleich mit der ortsüblichen Standardbehandlung. In kleineren Untersuchungen ohne Kontrollgruppen wurde die Zwangsbehandlung positiver bewertet, allerdings sind deren Resultate anfällig für Störgrössen und Fehlinterpretationen. Nachdenklich stimmen positive «Belege», wenn Betroffene überhaupt erst durch die Aufnahme in ein solches Programm eine angemessene psychiatrische Behandlung erhielten. Belegt und nicht weiter überraschend ist zudem, dass mit Zwangsmassnahmen in Berührung gekommene Personen in einer späteren Krise von sich aus weniger Hilfe suchen (Übersicht bei [17]).

\section{Kommentar}

Das neue ESR beansprucht, die Menschen zu schützen. Mit Blick auf die Psychiatrie stellt sich die Frage, 
wovor seelisch erkrankte Menschen geschützt werden müssen. Vor sich selbst, vor der Gesellschaft oder gar vor «der Psychiatrie»?

Obwohl das neue ESR psychiatriespezifische Passagen beinhaltet, ist wenig an fundamentaler Vorwärtsbewegung erkennbar. Der Gesetzgeber schliesst mit der Einführung von stationären Zwangsmassnahmen eine seiner Meinung nach bestehende «Gesetzeslücke», relativiert den Stellenwert psychiatrischer Patientenverfügungen und sieht erstmals ambulante Zwangsmassnahmen vor. Gegen diese nun schweizweit gesetzlich verankerte Diskriminie-
Im Kreislauf des Misstrauens geraten die Kliniken in eine juristische Absicherungsmentalität, indem sie mit einer kaum noch überblickbaren Flut an Formularen, Rechtsmittelbelehrungen und Unterschriften die Patienten und ihre Angehörigen permanent über ihre Rechte und behördlich-gerichtlichen Beschwerdemöglichkeiten aufklären. Doch statt dadurch entspannten Handlungsspielraum zu schaffen, steigert dieses Vorgehen das Misstrauen: Wer so viel auf Rechte und Beschwerdemöglichkeiten hinweisen muss, verfolgt kaum ehrbare Absichten. Unter dem neuen Gesetz geraten institutionell tätige Psychiater

\section{Der Gesetzgeber führt stationäre Zwangsmassnahmen ein, relativiert den Stellenwert psychiatrischer Patientenverfügungen und sieht erst- mals ambulante Zwangsmassnahmen vor.}

rung einer Bevölkerungsgruppe fallen der bereits bestehende gerichtliche Instanzenzug und positive Neuerungen wie etwa das Nennen einer Vertrauensperson oder Formvorschriften bei der FU wenig ins Gewicht. Letztlich handelt es sich dabei um Abwehrrechte gegen ein autoritäres Behandlungssystem, das weiterhin Menschen wegen einer seelischen Erkrankung in einer "geeigneten» Institution einschliesst, sie dort gegen ihren Willen behandelt und neu auch ambulante Massnahmen anordnet. Auftraggeber ist in diesen Fällen der Staat, nie sind es die Betroffenen selbst. Doch wie schützt man diese vor einem Staat, der selbst zu schützen vorgibt?

Mit der Einführung der psychiatriespezifischen Bestimmungen im neuen ESR nehmen Komplexität und Ausmass an staatlicher Kontrolle schwerer seelischer Erkrankungen zu. Psychiatrische Institutionen mit öffentlichem Versorgungsauftrag befinden sich in einer konflikthaften Double-bind-Situation. Einerseits werden sie von der Gesellschaft, die psychische Krankheit unter administrativer Kontrolle wissen will, zur Ausübung von Polizeifunktionen in einem Ausmass legitimiert und verpflichtet, das sie - von der Seuchenbekämpfung (Art. 15-17 EpG) einmal abgesehen - vom Rest der Medizin scharf trennt. Andererseits schlägt ihnen von derselben Gesellschaft tiefes Misstrauen entgegen, das sich in einer Unzahl von Vorschriften und Verboten manifestiert, mit denen ihr Tun diszipliniert werden soll. Wer mit derartiger Macht und Gewalt ausgestattet ist, bedarf seinerseits der rigorosen staatlichen Überwachung. Oder umgekehrt, aus Sicht der Psychiatrie: Die Verstaatlichung seelisch Erkrankter «zum Schutz ihrer Rechte und zum Schutz der Gesellschaft - in dieser Formel steckt das Misstrauen, das die Psychiater (...) hinderte, je problemlos liberal zu sein, und das sie als Vorkämpfer einer Politik staatlicher Eingriffe in eine Gesellschaft, die mit ihren Problemen nicht fertig wird, erscheinen» lässt [18]. bei der Behandlung von Menschen mit schweren seelischen Erkrankungen mehr denn je in die Rolle von Vollzugsbeauftragten einer staatlich regulierten Psychiatrie. Sie sollen angeordnete Therapien durchführen, Interventionen rapportieren und rechtfertigen, Behandlungen oder deren Abbruch behördlich genehmigen lassen, rückfallgefährdete Personen melden usw., die Liste liesse sich fortführen. Leidtragende sind Betroffene und Therapeuten gleichermassen: In einer formalisierten, von juristisch-behördlichen Denkschemata erfüllten Atmosphäre wird der Aufbau einer vertrauensvollen Beziehung, in der Zuversicht und Recovery gedeihen, mehr denn je zur Kunst.

Unsere Gesellschaft, die Autonomie und Freiheit hochhält, zeigt bisher wenig Toleranz gegenüber seelisch Erkrankten, die von der Norm abweichen und sich in der Öffentlichkeit «auffällig» verhalten. Das neue ESR setzt die Hürden für deren institutionelle Unterbringung nicht höher, etwas patientenfreundlichere Kriterien gelten einzig bei der (seltenen) Zurückbehaltung von freiwillig in die Klinik Eingetretenen. Ob deren Nachbarn in Zukunft mehr als bisher bereit sind, diese kleine Spitze des Eisbergs $\mathrm{zu}$ (er-)tragen, wird sich weisen.

Gegen die psychiatriespezifischen Teile der neuen gesetzlichen Bestimmungen wäre fachärztlicher Widerstand am Platz gewesen. Dass solcher nicht vernehmbar erfolgte, mag verschiedene Gründe haben. Einer davon ist, dass wir institutionell tätigen Psychiater uns seit langem daran gewöhnt haben, als verlängerter Arm eines «gutmeinenden», paternalistischen Staates bei seelisch Erkrankten Zwangsmassnahmen durchzuführen. Wir scheinen die Sichtweise über Zwang als notwendiges Übel internalisiert $\mathrm{zu}$ haben und finden unsererseits gute, heutzutage sogar neurobiologische Gründe, um Patienten «zu ihrem eigenen Wohl» gegen ihren Willen zu therapieren (s. beispielsweise das Konzept der «Neurotoxi- 
zität» unbehandelter Psychosen). Solch reduktionistische Begründungen sind allerdings gefährlich, da sie soziologische Komponenten von Erkrankungen und Krankheitskonzepten ausblenden und zudem ihrerseits das Risiko bergen, Betroffene gegen ihren Willen einer neurotoxischen Gefahr auszusetzen [19]. Die Vorbereitungsphase des neuen Gesetzes wäre ein günstiges Zeitfenster gewesen, über Funktionen einer zeitgemässen Psychiatrie nachzudenken, zugeschriebene Stereotype und Rollenerwartungen kritisch zu hinterfragen und den einen oder anderen gesellschaftlich zugedachten Auftrag zurückzuweisen. Das ist leider nicht geschehen.

\section{Institutionell tätige Psychiater geraten mehr denn je in die Rolle von Vollzugsbeauftragten einer staatlich regulierten Psychiatrie.}

Zusammenfassend lässt sich feststellen, dass das neue Gesetz für seelisch erkrankte Menschen kaum Fortschritt bringt. Vielmehr trifft, was Karl Kraus einst über die Psychoanalyse gesagt haben soll, auf das ESR zu: Es ist Teil des Problems, für dessen Lösung es sich hält.

\section{Literatur}

1 Schweizerisches Zivilgesetzbuch. www.admin.ch/ ch/d/sr/210/index.html, Online-Zugriff am 3.1.2013.

2 Reusser RE, Geiser T. Allgemeine Vorbemerkungen. In: Geiser T, Reusser RE (Hrsg.). Basler Kommentar Erwachsenenschutz. Basel: Helbing Lichtenhahn; 2012. S. 3-30.

3 Bridler R. Patientenverfügung und Zwangsernährung. Schweiz Ärztezeitung. 2011;92(19):714-7.

4 Gassmann J. Die Patientenverfügung. In: Rosch D, Büchler A, Jakob D (Hrsg.). Das neue Erwachsenenschutzrecht. Basel: Helbing Lichtenhahn; 2011. S. 78-90.

5 Botschaft zur Änderung des Schweizerischen Zivilgesetzbuches (Erwachsenenschutz, Personenrecht und Kindesrecht) vom 28. Juni 2006. p. 7030-4. www. admin.ch/ch/d/ff/2006/7001.pdf, Online-Zugriff am 16.1.2013.
6 Silveira MJ, Scott YHK, Langa KM. Advance directives and outcomes of surrogate decision making before death. N Engl J Med. 2010;362:1211-8.

7 Atkinson JM, Garner HC, Stuart S, Patrick H. The development of potential models of advance directives in mental health care. J Ment Health. 2003;12:575-84.

8 Huckshorn KA. Re-designing state mental health policy to prevent the use of seclusion and restraint. Adm Policy Ment Health. 2006;33(4):482-91.

9 Frajo-Apor B, Stippler M, Meise U. «Etwas Erniedrigenderes kann dir eigentlich in der Psychiatrie nicht passieren». Psychiat Prax. 2011;38:293-9.

10 Swanson JW, Swartz MS, Elbogen EB, Van Dorn RA, Ferron J, Wagner HR et al. Facilitated psychiatric advance directives: a randomized trial of an intervention to foster advance treatment planning among persons with severe mental illness. Am J Psychiatry. 2006;163:1943-51.

11 Reilly J, Atkinson JM. The content of mental health advance directives: advance statements in Scotland. Int J Law Psychiatry. 2010;33:116-21.

12 Srebnik DS, Rutherford LT, Peto T, Russo J, Zick E, Jaffe $\mathrm{C}$, et al. The content and clinical utility of psychiatric advance directives. Psychiatr Serv. 2005;56:592-98.

13 Papageorgiou A, King M, Janmohamed A, Davidson O, Dawson J. Advance directives for patients compulsorily admitted to hospital with serious mental disorders: directive content and feedback from patients and professionals. J Ment Health. 2004;13:379-88.

14 Reusser RE, Geiser T. Allgemeine Vorbemerkungen. In: Geiser T, Reusser RE (Hrsg.). Basler Kommentar Erwachsenenschutz. Basel: Helbing Lichtenhahn; 2012. S. 3-30.

15 Botschaft des Bundesrates zur Änderung des Schweizerischen Zivilgesetzbuches (Erwachsenenschutz, Personenrecht und Kindesrecht) vom 28. Juni 2006.

16 Geiser T, Etzensberger M. Die Fürsorgerische Unterbringung. In: Geiser T, Reusser RE (Hrsg.). Basler Kommentar Erwachsenenschutz. Basel: Helbing Lichtenhahn; 2012. S. 444-529.

17 Bridler R, Gassmann J. Die Praxis der ambulanten Zwangsbehandlung in anderen Ländern. Schweiz Ärztezeitung. 2010; 91(45):1790-94.

18 Dörner K. Bürger und Irre. Hamburg: Europäische Verlagsanstalt; 1999.

19 Ho BC, Andreasen NC, Ziebell S, Pierson R, Magnotta V. Long-term antipsychotic treatment and brain volumes - a longitudinal study of first-episode schizophrenia. Arch Gen Psychiatry. 2011;68(2): 128-37. 